\title{
Asymptotically optimal pairing strategy for Tic-Tac-Toe with numerous directions
}

\author{
Padmini Mukkamala \\ Rutgers, New Jersey \\ Dömötör Pálvölgyi \\ Eötvös University, Budapest \\ Submitted: May 29, 2010; Accepted: Sep 19, 2010; Published: Oct 15, 2010 \\ Mathematics Subject Classification: 91A46
}

\begin{abstract}
We show that there is an $m=2 n+o(n)$, such that, in the Maker-Breaker game played on $\mathbb{Z}^{d}$ where Maker needs to put at least $m$ of his marks consecutively in one of $n$ given winning directions, Breaker can force a draw using a pairing strategy. This improves the result of Kruczek and Sundberg [15] who showed that such a pairing strategy exists if $m \geqslant 3 n$. A simple argument shows that $m$ has to be at least $2 n+1$ if Breaker is only allowed to use a pairing strategy, thus the main term of our bound is optimal.
\end{abstract}

\section{Introduction}

A central topic of combinatorial game theory is the study of positional games, the interested reader can find the state of the art methods in Beck's Tic-Tac-Toe book [4]. In general, positional games are played between two players on a board, the points of which they alternatingly occupy with their marks and whoever first fills a winning set completely with her/his marks wins the game. Thus a positional game can be played on any hypergraph, but in this paper, we only consider semi-infinite games where all winning sets are finite. If after countably many steps none of them occupied a winning set, we say that the game ended in a draw. It is easy to see that we can suppose that the next move of the players depends only on the actual position of the board and is deterministic. ${ }^{1}$ We say that a player has a winning strategy if no matter how the other player plays, she/he always wins. We also say that a player has a drawing strategy if no matter how the other player plays, she/he can always achieve a draw (or win). A folklore strategy stealing argument shows that the second player (who puts his first mark after the first player puts her first mark, as ladies go first) cannot have a winning strategy, so the best that he can

\footnotetext{
${ }^{1}$ This is not the case for infinite games and even in semi-infinite games it can happen that the first player can always win the game but there is no $N$ such that the game could be won in $N$ moves. For interesting examples, we refer the reader to the antique papers $[2,5,7]$.
} 
hope for is a draw. Given any semi-infinite game, either the first player has a winning strategy, or the second player has a drawing strategy. We say that the second player can achieve a pairing strategy draw if there is a matching among the points of the board such that every winning set contains at least one pair. It is easy to see that the second player can now force a draw by putting his mark always on the point which is matched to the point occupied by the first player in the previous step (or anywhere, if the point in unmatched). Note that in a relaxation of the game for the first player, by allowing her to win if she occupies a winning set (not necessarily first), the pairing strategy still lets the second player to force a draw. Such drawing strategies are called strong draws. Since in these games only the first player is trying to complete a winning set and the second is only trying to prevent her from doing so, in these games, the first player is called Maker, the second Breaker, and the game is called a Maker-Breaker game.

This paper is about a generalization of the Five-in-a-Row game ${ }^{2}$ which is the more serious version of the classic Tic-Tac-Toe game. This generalized game is played on the $d$-dimensinal integer grid, $\mathbb{Z}^{d}$, and the winning sets consist of $m$ consecutive gridpoints in $n$ previously given directions. For example, in the Five-in-a-Row game $d=2, m=5$ and $n=4$, the winning directions are the vertical, the horizontal and the two diagonals with slope 1 and -1 . Note that we only assume that the greatest common divisor of the coordinates of each direction is 1 , so a direction can be arbitrarily long, e.g. $(5,0,24601)$. The question is, for what values of $m$ can we guarantee that the second player has a drawing strategy? It was shown by Hales and Jewett [4], that for the four above given directions of the two dimensional grid and $m=9$ the second player can achieve a pairing strategy draw. In the general version, a somewhat weaker result was shown by Kruczek and Sundberg [15], who showed that the second player has a pairing strategy if $m \geqslant 3 n$ for any $d$. They conjectured that there is always a pairing strategy for $m \geqslant 2 n+1$, generalizing the result of Hales and Jewett. ${ }^{3}$

Conjecture 1 (Kruczek and Sundberg). If $m=2 n+1$, then in the Maker-Breaker game played on $\mathbb{Z}^{d}$, where Maker needs to put at least $m$ of his marks consecutively in one of $n$ given winning directions, Breaker can force a draw using a pairing strategy.

Our main result asymptotically solves their conjecture.

Theorem 2. There is an $m=2 n+o(n)$ such that in the Maker-Breaker game played on $\mathbb{Z}^{d}$, where Maker needs to put at least $m$ of his marks consecutively in one of $n$ given winning directions, Breaker can force a draw using a pairing strategy.

In fact we prove the following theorem, which is clearly stronger because of the classical result [10] showing that there is a prime between $n$ and $n+o(n)$.

Theorem 3. If $p=m-1 \geqslant 2 n+1$ is a prime, then in the Maker-Breaker game played on $\mathbb{Z}^{d}$, where Maker needs to put at least $m$ of his marks consecutively in one of $n$ given winning directions, Breaker can force a draw using a pairing strategy.

\footnotetext{
${ }^{2}$ Aka Go-Muku and Amőba.

${ }^{3}$ It is not hard to show that if $m=2 n$, then such a strategy might not exist, we show why in Section 3.
} 
The proof of the theorem is by reduction to a game played on $\mathbb{Z}$ and then using the following recent number theoretic result of Preissmann and Mischler. Later this result was independently rediscovered by Kohen and Sadofschi [13] and by Karasev and Petrov [11], they both gave a short proof using the Combinatorial Nullstellansatz [1]. The latter paper also gives an even shorter topological proof and generalizations.

Lemma 4. [17] Given $d_{1}, \ldots, d_{n}$ and $p \geqslant 2 n+1$ prime, we can select $2 n$ numbers, $x_{1}, \ldots, x_{n}, y_{1}, \ldots, y_{n}$ all different modulo $p$ such that $x_{i}+d_{i} \equiv y_{i} \bmod p$.

We prove our theorem in the next section and end the paper with some additional remarks.

\section{Proof of Theorem 3}

We consider the winning directions to be the primivite vectors ${ }^{4} \vec{v}_{1}, \ldots, \vec{v}_{n}$. Using a standard compactness argument it is enough to show that there is a pairing strategy if the board is $[N]^{d}$, where $[N]$ stands for $\{1, \ldots, N\}$. For interested readers, the compactness argument is discussed in detail at the end of this section.

First we reduce the problem to one dimension. Take a vector $\vec{r}=\left(r_{1}, r_{2}, \ldots, r_{d}\right)$ and transform each grid point $\vec{v}$ to $\vec{v} \cdot \vec{r}$. If $\vec{r}$ is such that $r_{j}>0$ and $r_{j+1}>N\left(r_{1}+\ldots+r_{j}\right)$ for all $j$, then this transformation is injective from $[N]^{d}$ to $\mathbb{Z}$ and each winning direction is transformed to some number, $d_{i}=\left|\vec{r} \cdot \vec{v}_{i}\right|{ }^{5}$ So we have these $n$ differences, $d_{1}, \ldots, d_{n}$, and the problem reduces to avoiding arithmetic progressions of length $m$ with these differences. From the reduction it follows that if we have a pairing strategy for this game, we also have one for the original.

Let $p$ be a prime such that $2 n+1 \leqslant p \leqslant 2 n+1+o(n)$. (In [10] it was shown that we can always find such a $p$ ). If we pick a vector $\vec{u}$ uniformly at random from $[p]^{d}$, then for any primitive vector $\vec{v}, \vec{u} \cdot \vec{v}$ will be divisible by $p$ with probability $1 / p$. Since each winning direction was a primitive vector, using the union bound, the probability that at least one of the $\vec{u} \cdot \vec{v}_{i}$ is divisible by $p$ is at most $n / p<1 / 2$. So, there is a $\vec{u}^{\prime}=\left(u_{1}^{\prime}, u_{2}^{\prime}, . ., u_{d}^{\prime}\right) \in[p]^{d}$ such that none of $\vec{u}^{\prime} \cdot \vec{v}_{i}$ is divisible by $p$. If we now take $\vec{r}=\left(r_{1}, r_{2}, . ., r_{d}\right)$ such that $r_{j}=u_{j}^{\prime}+(p N)^{j-1}$, then the dot product with $\vec{r}$ is injective from $[N]^{d}$ to $\mathbb{Z}$ and none of the $d_{i}=\vec{r} \cdot \vec{v}_{i}$ are divisible by $p$, since $\forall j r_{j} \equiv u_{j}^{\prime} \bmod p$.

We now apply Lemma 4 for $d_{1}, \ldots, d_{n}$ to get $2 n$ distinct numbers $x_{1}, x_{2}, \ldots x_{n}, y_{1}, y_{2}, . ., y_{n}$ such that $0 \leqslant x_{i}, y_{i}<p$ and $x_{i}+d_{i} \equiv y_{i} \bmod p$. Our pairing strategy is, for every $x \equiv x_{i}$ $\bmod p, x$ is paired to $x+d_{i}$ and if $x \equiv y_{i} \bmod p$, then $x$ is paired to $x-d_{i}$.

To see that this is a good pairing strategy, consider an arithmetic progression $a_{1}, \ldots, a_{m}$ of $m=p+1$ numbers with difference, say, $d_{i}$. Since $p$ and $d_{i}$ are coprimes, one of the numbers $a_{1}, \ldots, a_{m-1}$, say $a_{j}$, must be such that $a_{j} \equiv x_{i} \bmod p$. Hence $a_{j}, a_{j+1}$ must be paired in our pairing strategy, showing both cannot be occupied by Maker.

\footnotetext{
${ }^{4} \mathrm{~A}$ vector $\left(v_{1}, \ldots, v_{d}\right) \in \mathbb{Z}^{d}$ is primitive if $\operatorname{gcd}\left(v_{1}, \ldots, v_{d}\right)=1$.

${ }^{5}$ It is even possible that some of these numbers are zero, we will take care of this later.
} 
For completeness here we sketch how the compactness argument goes. We show that it is sufficient to show that a pairing strategy exists for every finite $[N]^{d}$ board. For this we use the following lemma. ${ }^{6}$

Lemma 5. [14] (König's Infinity Lemma) Let $V_{0}, V_{1},$. be an infinite sequence of disjoint non-empty finite sets, and let $G$ be a graph on their union. Assume that every vertex $v$ in a set $V_{N}$ with $N \geqslant 1$ has a neighbour $f(v)$ in $V_{N-1}$. Then $G$ contains an infinite path, $v_{0} v_{1} \ldots$ with $v_{N} \in V_{N}$ for all $N$.

Given a pairing strategy for $\left[N_{0}\right]^{d}$, consider a smaller board $[N]^{d}$ where $N<N_{0}$. We can think of a pairing strategy as, essentially, a partition of $\left[N_{0}\right]^{d}$ into pairs and unpaired elements. $^{7}$ We can construct a good pairing strategy for the smaller board by taking the restriction of these set of pairs to $[N]^{d}$ and leave the elements paired outside $[N]^{d}$ as unpaired elements. We call this as a restriction of the pairing strategy to the new board. As long as we do not change the length of the winning sets and the prescribed directions, any winning set in the $[N]^{d}$ board is also a winning set in the $\left[N_{0}\right]^{d}$ board and hence must have a pair from the restriction. Hence, the Breaker can block all winning pairs and the restriction of the pairing strategy is a valid strategy for Breaker for the smaller board.

We can now prove the following theorem,

Theorem 6. Given a fixed set $S,|S|=n$, of winning directions, and positive integer $m$, if Breaker has a pairing strategy for all boards $[N]^{d}$ and length of winning sets equal to $m$, then Breaker also has a pairing strategy for the $\mathbb{Z}^{d}$ board.

We will apply König's Infinity Lemma to prove the theorem. Let $V_{N}$ be the set of all pairing strategies on the $\{-N, \ldots, N\}^{d}$ board with winning sets as defined in the theorem. We say a strategy in $V_{N-1}$ and a strategy in $V_{N}$ have an edge between them if the former is a restriction of the latter. It is easy to see that every vertex in $V_{N}$ does have an edge to its restriction in $V_{N-1}$. Hence, by the lemma, we must have an infinite path $v_{0} v_{1} \ldots$. The union of all these pairing strategies gives a valid pairing strategy for the infinite game.

\section{Possible further improvements and remarks}

As we said before, if $m \leqslant 2 n$, then the second player cannot have a pairing strategy draw. This can be seen as follows. On one hand, in any pairing strategy, from any $m$ consecutive points in a winning direction, there must be at least two points paired to each other in this direction. On the other hand, there must be a winning direction in which at most $1 / n$ of all points are matched to another in this direction. If we pick a set of size $m-1$ uniformly randomly in this direction, then the expected number of points mathced in this direction will be at most $(m-1) / n<2$. Thus, there is a set of size $m-1$ that contains

\footnotetext{
${ }^{6}$ We use the version stated in [8].

${ }^{7}$ Note that a pairing strategy does not guarantee that every element is paired. It only states that every winning set has a pair. Hence there might be many unpaired elements in a pairing strategy.
} 
only one such point. Its matching point can now be avoided by extending this set to one way or the other, thereby giving us a winning set with no matched pair.

If $n=1$ or 2 , then a not too deep case analysis shows that the first player has a winning strategy if $m=2 n$, even in the strong game, where the second player also wins if he occupies a winning set. Moreover, the second player has a pairing strategy for $m=2 n+1$ if $n=1$ or 2 , thus, in this case, the conjecture is tight. However, for higher values, it seems that Breaker can always do better than just playing a pairing strategy, so we should not expect this strategy the best to achieve a draw. Quite tight bounds have been proved for Maker-Breaker games with potential based arguments, for the latest in generalization of Tic-Tac-Toe games, see [16]. Despite this, from a combinatorial point of view, it still remains an interesting question to determine the best pairing strategy. Unfortunately our proof can only give $2 n+2$ (if $2 n+1$ is a prime) which is still one bigger than the conjecture.

One could hope that maybe we could achieve a better bound using a stronger result than Lemma 4 (see for example the conjecture of Roland Bacher in [17], whom we would like to thank for directing us to it [3]), however, already for $n=3$, our method cannot work. Consider the three directions $(1,0),(0,1),(1,1)$. Optimally, we would hope to map them to three numbers, $d_{1}, d_{2}, d_{3}$, all coprime to 6 , such that we can find $x_{1}, x_{2}, x_{3}, y_{1}, y_{2}, y_{3}$ all different modulo 6 such that $x_{i}+d_{i} \equiv y_{i} \bmod 6$. But this is impossible since $d_{3}=$ $d_{1}+d_{2}$, so we cannot even fulfill the condition that the differences have to be coprimes to 6. But even if we forget about that condition, it would still be impossible to find a triple satisfying $d_{3}=d_{1}+d_{2}$. If we consider a pairing strategy where the pair of any grid point $\vec{v}$, depends only on $v \cdot r$, then the above argument shows that such a pairing strategy does not exist for the three vectors $(1,0),(0,1),(1,1)$. However, it is not hard to find a suitable periodic pairing strategy for these three vectors. We would like to end with an equivalent formulation of Conjecture 1.

Conjecture 7 (Kruczek and Sundberg, reformulated). Suppose we are given $n$ primitive vectors, $\vec{v}_{i}$ of $\mathbb{Z}_{2 n}^{d}$ for $i \in[n]$. Is it always possible to find a partition of $\mathbb{Z}_{2 n}^{d}$ into $\vec{x}_{i}^{j}, \vec{y}_{i}^{j}$ for $i \in[n], j \in[2 n]$ such that $\vec{x}_{i}^{j}+\vec{v}_{i}=\vec{y}_{i}^{j}$ and $\vec{x}_{i}^{j}-\vec{x}_{i}^{j^{\prime}}$ is not a multiple of $\vec{v}_{i}$ for $j \neq j^{\prime}$ ?

Also, one can formulate a more daring conjecture about general graphs.

Conjecture 8. Suppose that the edges of a $2 d$-regular graph are colored such that the edges of each color form a cycle of length $2 d$. Then there is a perfect matching containing one edge of each color.

For $d=2$, there is a simple proof by Zoltán Király [12], who also invented the above formulation of the problem. However, since the submission of our paper, this conjecture was disproved on the first Emléktábla Workshop [6]. It was shown with a tricky construction that maybe there is no perfect matching at all, yet alone a rainbow one. They asked whether the conjecture holds for bipartite graphs, in which case the existence of a (non-rainbow) matching is guaranteed by Hall's theorem [10]. 


\section{Acknowledgment}

We are very thankful to the anonymous referee for her/his helpful observations.

\section{References}

[1] N. Alon: Combinatorial Nullstellensatz, Combinatorics, Prob. and Comp. 8 (1-2) (1999) 7-29.

[2] M.Ajtai, L. Csirmaz, Zs.Nagy: On a generalization of the game go-moku, I Studia Mathematica 14 (1979), pp. 209-226.

[3] Roland Bacher, http://mathoverflow.net/questions/24108/ can-select-many-disjoint-pairs-with-prescribed-differences-from-z-n

[4] J. Beck: Combinatorial games: tic-tac-toe theory. Cambridge University Press, 2008.

[5] J. Beck, L. Csirmaz: Variations on a game, Journal of Combinatorial Theory, Series A (33) 1982, pp. 297-315.

[6] Fabricio Benevides, Michal Przykucki, Tamás Terpai, Mykhaylo Tyomkyn, personal communication. See also http://www.renyi.hu/ emlektab/

[7] L. Csirmaz, Zs. Nagy: On a generalization of the game go-moku, II Studia Mathematica 14 (1979) pp. 461-469.

[8] R. Diestel: Graph Theory (Third Edition), Graduate Texts in Mathematics 173 (2005) pp. 200.

[9] P. Hall: On Representatives of Subsets, J. London Math. Soc. 10, 26-30, 1935.

[10] G. Hoheisel: Primzahlenprobleme in der Analysis, Sitzungber. Berlin Math. Ges. (1930), 550-558.

[11] R.N. Karasev, F.V. Petrov: Partitions of nonzero elements of a finite field into pairs, http://arxiv.org/abs/1005.1177

[12] Z. Király, personal communication.

[13] D. Kohen and I. Sadofschi: A New Approach on the Seating Couples Problem, http: //arxiv.org/abs/1006.2571

[14] D. König: Theorie der Endlichen und Unendlichen Graphen, Kombinatorische Topologie der Streckenkomplexe, Leipzig, Akad. Verlag.

[15] K. Kruczek and E. Sundberg, A Pairing Strategy for Tic-Tac-Toe on the Integer Lattice with Numerous Directions. Electronic Journal of Combinatorics 15(1):N42, 2008.

[16] K. Kruczek and E. Sundberg, Potential-Based Strategies for Tic-Tac-Toe on the Integer Lattice with Numerous Directions. Electronic Journal of Combinatorics 17(1):R5, 2010.

[17] Emmanuel Preissmann and Maurice Mischler, Seating Couples Around the King's Table and a New Characterization of Prime Numbers, American Mathematical Monthly, Vol. 116, No. 3, 2009, pp. 268-272. 